\title{
Waiting Time Estimates in Symmetric ATM-Oriented Rings with the Destination Release of Used Slots
}

\author{
Mirjana Zafirovic-Vukotic, Senior Member, IEEE, and Ignatius G. M. M. Niemegeers, Member, IEEE
}

\begin{abstract}
This paper considers the basic access mechanism in asynchronous transfer mode (ATM)-oriented rings which, like Orwell, ATM ring, and ALine, apply destination release of used slots. The released slots may be reused by the destination station, or in some protocols, they must be given to the next station. Such a mechanism can be modeled by a multiple-server multiqueue system, where switchover times between consecutive polling instants of the queues are nonzero. The server polls the queues according to a certain polling discipline, which is dependent on the service time. This paper presents approximate mean minipacket waiting times in such systems. The approximation is based on a polling queueing model with Markovian server routing. Poisson arrivals and a symmetric workload model for minipackets having a full and partial traffic matrix are assumed. The performance characteristics are compared between the alternative options.
\end{abstract}

Index Terms-ATM, LAN, queueing model.

\section{INTRODUCTION}

$\mathbf{T}$ HIS PAPER deals with a slotted-ring basic-access mechanism in high-speed local area networks (LAN's), which are asynchronous transfer mode (ATM)-oriented. In particular, only mechanisms which apply the destination release of used slots, like ATM rings (ATMR 's) [1], Orwell [2], and ALine [10], are considered.

The basic access mechanism in such rings is as follows. The ring is partitioned into equal-length slots, as illustrated in Fig. 1. Slots circulate around the ring and can be empty or full. A full slot is occupied by a minipacket, i.e., a medium access control protocol data unit (MAC PDU), which may include an ATM cell. A full slot circulating around the ring reaches the destination station, which then reads it and passes it on to a higher layer. We assume that each station is capable of using every empty slot that arrives, and reading each slot destined to itself.

There are two variants regarding the slot reuse.

Case N: The destination station releases a slot that was full as an empty slot and the next downstream station may use it (Case $N, N$ denoting no slot reuse).

Case $Y$ : The destination station may reuse the slot that was full for its own transmission (Case $Y, Y$

Manuscript received March 24, 1997; revised June 9, 1998; approved by IEEE/ACM TRANSACTIONS ON NETWORKING Editor P. O'Reilly.

M. Zafirovic-Vukotic is with the M. Pupin Intitute, Telecommunications Department, University of Belgrade, 11000 Beograd, Yugoslavia (e-mail: ezafirov@ubbg.etf.bg.ac.yu).

I. G. M. M. Niemegeers is with CTIT, University of Twente, 7500 AE Enschede, The Netherlands (e-mail: niemegee@cs.utwente.nl).

Publisher Item Identifier S 1063-6692(99)03822-4.

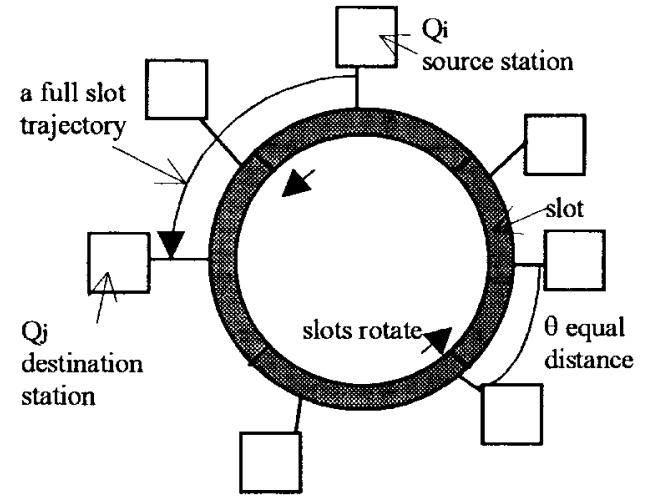

Fig. 1. A full-slot trajectory in an ATM-oriented ring with the destination release of used slots.

denoting yes for the slot reuse). Note that protocols [1], [2], and [10] apply slot reuse by destination (Case $Y$ ). The older variant of the Orwell protocol corresponded to Case $N$ [7].

Modeling of the basic access mechanism in ATM-oriented rings with the destination release of used slots in Case $N$ has been done in [7] as follows. It has been shown that such a mechanism can be modeled by a multiple-server multiqueue system, where switchover times between consecutive polling instants of the queues are nonzero. The service strategy at a queue is 1-limited. The server polls the queues according to a certain polling discipline, which is dependent on the service time. The Markovian server polling model to approximate such a system was stated in [7], and the exact stability conditions for a symmetric case derived from it. However, no attempt to solve this model as a polling model was made. Instead, a service preemptive model with minipacket batch arrivals leads to the delay estimates. Another effort to estimate the delays in the ATM-oriented ring with the destination release of used slots was done in [9], and an evaluation of the estimate is given in Section VI-C.

The waiting time approximation in this paper is based on a polling model with Markovian server routing, 1-limited service discipline and Poisson arrival process. The pseudoconservation law for this case is derived in [6]. It gives a weighted sum of the expected customer waiting times, and for a symmetric case, it gives the expected waiting times. A possibility of using the Markovian server routing model as solved in [6] for approximate Orwell modeling Case $N$ is mentioned in a sentence in [6], even though the idea was not elaborated on in any way. 
We follow the Markovian server polling model approach, and that is the contribution of [7] to this paper. The idea of using a polling model with Markovian server routing to represent Case $Y$ is new. We apply the solution of the model given in [6] which has not been done up to now, and we obtain the delay estimates and stability conditions for the ATMR in diverse cases. Two traffic cases are considered: 1) a full (Case $F$ ) and 2) a partial traffic matrix case (Case $P$ ). In the full traffic matrix case, a station sends minipackets to itself, whereas in the partial traffic matrix case, it does not.

In addition, a cyclic and a random polling model are used in this paper. The cyclic model evaluates the lower boundary for the expected delays and provides an estimate of the expected delays under a low load. Mean waiting times in single-server cyclic polling systems have been obtained for symmetric and some asymmetric cases, e.g., see [3] and [5]. An analysis of single-server random polling systems, where each queue is polled with a certain probability, has been done in [4]. Formulas for mean waiting times have been derived for a fully symmetric case, and a unique service strategy is used at all queues. The random polling model could provide estimates of the expected delays under high loads in ATMRs for some cases.

The organization of the rest of the paper is as follows. The general model of the basic access mechanism in the ATMoriented rings is presented in Section II. Section III presents approximate modeling for a single-slot ring. The exact stability condition is stated, and approximate mean waiting time models for high-load and low-load intensity are derived in Section IV. Approximate minipacket waiting time modeling for a multipleslot case is done in Section V. The model is tested and the performance characteristics between the protocol options are compared in Section VI. The conclusions are presented in Section VII.

\section{GENERAL MODEL}

\section{A. Assumptions and Notation}

We consider performance modeling of the basic access mechanism in a fully symmetric ATMR and under a fully symmetric workload.

The ring is partitioned into $m$ slots. The slot duration $\sigma$ equals the number of bits in the slot divided by the transmission rate. Therefore, the total propagation time in the ring $\tau$, i.e., a slot rotation time, equals

$$
\tau=m \sigma \text {. }
$$

The ATMR has $n$ stations (queues) $Q_{1}, \cdots, Q_{n}$, where each station has an infinite buffer capacity to store waiting minipackets (customers). We assume that the distance between the consecutive stations is equal and the signal propagation time between the two consecutive stations equals $\theta$. The total propagation time in the ring $\tau$ equals

$$
\tau=n \theta \text {. }
$$

Customers arriving at queue $Q_{i}$ are called type- $i$ customers. Customers arrive at $Q_{i}$ according to the independent Poisson processes with arrival intensity $\lambda$.
In this paper, we consider two traffic cases: 1) a full (Case $F$ ) and 2) a partial (Case $P$ ) traffic matrix case. In the full traffic matrix case, each minipacket has destination $Q_{j}$, with probability $1 / n$. In the partial traffic matrix case, each minipacket which originated at $Q_{i}$ has destination $Q_{j}$, with the probability $1 /(n-1)$ if $i \neq j$, and zero if $i=j$. So, in the full traffic matrix case, a station may send minipackets to itself, whereas in the partial traffic matrix case it may not. In reality, a station sends to itself just the broadcast data, some control and management messages, while other data which actually constitutes the largest part of the network traffic is not sent to itself. Therefore, Case $P$ is slightly more realistic than Case F.

\section{B. The Queueing Model}

The queues are polled by multiple servers, each corresponding to a slot. When the queue polled $Q_{i}$ is empty, the server $S$ polls the next queue $Q_{i+1}$. Otherwise, $S$ serves according to a 1-limited service discipline, i.e., it serves only one type- $i$ customer at $Q_{i}$. The order of service within each queue is on a first-come-first-serve basis. This assumption is not essential, and this model holds for a priority case, as well.

The switchover time of the server between the previously polled queue and $Q_{i}$ is a constant and equals $\theta$.

A customer's service corresponds to the transfer of a minipacket in a slot from $Q_{i}$ to $Q_{j}$. The service time corresponds to the propagation time from the source $Q_{i}$ to the destination $Q_{j}$. The service time is an integer multiple of $\theta$ and it equals $k \theta$, in Case $N$, being the distance between $Q_{i}$ and $Q_{j}$, i.e., $k=j-i$, for $j>i$. In Case $Y$, the service time equals $(k-1) \theta$, accepting a constant switchover time in the model and equal to $\theta$. After that service, $S$ polls the next queue after the destination, $Q_{j+1}$, in Case $N$ or it polls $Q_{j}$ in Case $Y$. Thus, the polling discipline is dependent on the service time.

Further, the service times of type- $i$ customers are independent, identically distributed stochastic variables $B$ with the following distribution, and the first moment $\beta$ and the second moment $\beta^{(2)}$.

Case $F$ and Case $N-$ Subscript $F N$ :

$$
\begin{aligned}
P\left\{B_{F N}=k \theta\right\} & =1 / n, \quad k=1,2, \cdots, n \\
\beta_{F N} & =\frac{(n+1) \tau}{2 n} \\
\beta_{F N}^{(2)} & =\frac{(n+1)(2 n+1) \tau^{2}}{6 n^{2}} .
\end{aligned}
$$

Case $P$ and Case $N-$ Subscript PN:

$$
\begin{aligned}
P\left\{B_{P N}=k \theta\right\} & =1 /(n-1), \quad k=1,2, \cdots, n-1 \\
\beta_{P N} & =\frac{\tau}{2} \\
\beta_{P N}^{(2)} & =\frac{(2 n-1) \tau^{2}}{6 n} .
\end{aligned}
$$

Case $F$ and Case $Y$-Subscript FY:

$$
\begin{aligned}
P\left\{B_{F Y}=(k-1) \theta\right\} & =1 / n, \quad k=1,2, \cdots, n \\
\beta_{F Y} & =\frac{(n-1) \tau}{2 n} \\
\beta_{F Y}^{(2)} & =\frac{(n-1)(2 n-1) \tau^{2}}{6 n^{2}} .
\end{aligned}
$$




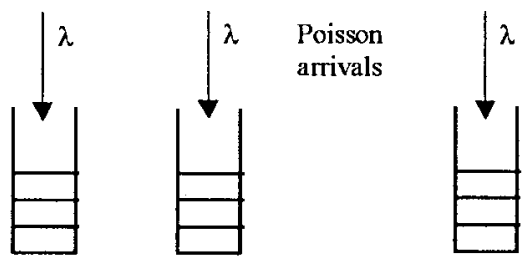

Markovian server routing

service time: $\beta, \beta$

Fig. 2. The Markovian server routing model for ATMR's.

Case $P$ and Case $Y$-Subscript PY:

$P\left\{B_{P Y}=(k-1) \theta\right\}=1 /(n-1), \quad k=1,2, \cdots, n-1$

$$
\begin{aligned}
& \beta_{P Y}=\frac{(n-2) \tau}{2 n} \\
& \beta_{P Y}^{(2)}=\frac{(n-2)(2 n-3) \tau^{2}}{6 n^{2}} .
\end{aligned}
$$

The offered traffic at $Q_{i}, \rho$, is defined as

$$
\rho=\lambda \beta .
$$

This description of the multiqueue multiple-server model accurately corresponds to a single- and a multiple-slot ring $(m \geq 1)$. Unfortunately, this model cannot be solved. In the remainder of this paper, first we will consider the single-slot case and provide an approximate solution to it (Section III). Then we will extend that approximate solution to a multipleslot case (Section V).

\section{ApProximate Modeling: Single-Slot RinG}

In this section, let us consider a single-slot ATMR. We have a single-server multiqueue polling model, as illustrated in Fig. 2. Since $m=1,(1)$ and (2) become

$$
\begin{aligned}
& \tau=\sigma \\
& \sigma=n \theta .
\end{aligned}
$$

The polling discipline in the multiqueue model of an ATMR is dependent on the service time, as shown in Section II. For the moment we assume that it is not. We use a polling model with Markovian server routing.

Let us at first consider the full traffic matrix case (superscript $F$ ) and the partial traffic matrix case with no slot reuse (superscript $P N$ ). The server, which corresponds to a slot, on departure from $Q_{i}$ visits $Q_{j}$ with probability $p_{i j}$, which is defined to have the following values:

$$
\begin{aligned}
p_{i j}^{F, P N} & = \begin{cases}a, & j=i+1 \\
b, & j \neq i+1\end{cases} \\
a+(n-1) b & =1, \quad a, b>0 .
\end{aligned}
$$

In the remainder of this paper, the system is assumed to be in equilibrium. Let us now define $\psi$ as the probability that the queue to which the service is offered is not empty. Due to the work balance argument, $\psi$ also equals the mean number of minipacket arrivals to $Q_{i}$ between two successive visits (and potential services) of the server at $Q_{i}$. Clearly, since the system is in equilibrium, we have $0<\psi<1$.

An empty slot visits the next downstream station with probability $a$, and every other station with probability $b$ after the departure from $Q_{i}$. If $Q_{i}$ did not use the slot but left it empty, the slot visits the next downstream station $Q_{i+1}$. An empty slot visits some station other than $Q_{i+1}$ only if $Q_{i}$ released a full slot. Following that reasoning, we have

$$
\begin{aligned}
p_{i j}^{F} & = \begin{cases}1-\psi+\frac{1}{n} \psi, & j=i+1 \\
\frac{1}{n} \psi, & j \neq i+1\end{cases} \\
p_{i j}^{P N} & = \begin{cases}1-\psi, & j=i+1 \\
\frac{1}{n-1} \psi, & j \neq i+1\end{cases}
\end{aligned}
$$

with $0<\psi<1$.

Let us now consider the partial traffic matrix case with slot reuse (superscript $P Y$ ). The server, which corresponds to a slot, on departure from $Q_{i}$ visits $Q_{j}$ with probability $p_{i j}$, which has the following values

$$
\begin{array}{r}
p_{i j}^{P Y}= \begin{cases}a, & j=i+1 \\
b, & j \neq i+1 \\
0, & j=i\end{cases} \\
a+(n-2) b=1, \quad a, b>0 .
\end{array}
$$

An empty slot visits the next downstream station with probability $a$, and some other station except itself with probability $b$, after the departure from $Q_{i}$. It never visits station $Q_{i}$ again right after the departure from it, in Case PY. Following that reasoning, we have

$$
p_{i j}^{P Y}= \begin{cases}1-\psi+\frac{1}{n-1} \psi, & j=i+1 \\ \frac{1}{n-1} \psi, & j \neq i+1 \\ 0, & j=i\end{cases}
$$

with $0<\psi<1$.

The expected time $E T_{i i}$ between two successive visits (and potential services) of the server at $Q_{i}$ is given by (A.14), which is derived from the underlying reversed Markov chain of the departure process, as presented in the Appendix. The mean number of minipacket arrivals between two successive visits $\psi$ is determined simply by Little's law, so multiplying the mean arrival rate $\lambda$ by the expected time $E T_{i i}$ (A.14), cf. (15)

$$
\psi=\lambda \frac{\sigma}{1-n \lambda \beta} .
$$

Due to the definition of $\psi$, we have $0<\psi<1$, as already explained. Note that because of (25), the stability condition [see (49)] leads to the relation $0<\psi<1$, as well.

Parameter $b$ equals [see (18)-(24)]

$$
\begin{aligned}
& b_{F}=\frac{1}{n} \psi_{F} \\
& b_{P}=\frac{1}{n-1} \psi_{P} .
\end{aligned}
$$


We get the following values for $b$ in the four cases (which are denoted by $F N, P N, F Y$, and $P Y$ ), taking $\beta$ from (4), (7), (10), (13), and using (16) and (25)-(27)

$$
\begin{aligned}
b_{F N} & =\frac{2 \lambda \sigma}{n(2-(n+1) \lambda \sigma)} \\
b_{P N} & =\frac{2 \lambda \sigma}{(n-1)(2-n \lambda \sigma)} \\
b_{F Y} & =\frac{2 \lambda \sigma}{n(2-(n-1) \lambda \sigma)} \\
b_{P Y} & =\frac{2 \lambda \sigma}{(n-1)(2-(n-2) \lambda \sigma)} .
\end{aligned}
$$

The pseudo-conservation law for the Markovian server routing model [6] leads to the expected minipacket waiting time $E W$, as follows:

$$
E W=\frac{n \lambda \beta^{(2)}+n \theta \rho+\theta}{2(1-n \rho-n \lambda \theta)}+\frac{1-n \rho}{n(1-n \rho-n \lambda \theta)} \sum_{\substack{k=1 \\ k \neq i}}^{n} E T_{k i}
$$

where $E T_{i j}:=E\{$ time between a departure of slot/server $S$ from $Q_{j}$ and its last departure from $\left.Q_{i}\right\}$.

The underlying reversed Markov chain of the departure process is used in the Appendix to derive an expression for $E T_{i j}$, see (A.10), in terms of $\sum y_{j}$, where $y_{j}$ is the expected number of steps between two successive server visits to $Q_{j}$. An expression for $\sum y_{j}$ is given by (A.20). Substituting (A.10) into (32), and taking (15) and (17), we get

$E W=\frac{1}{1-n \lambda \beta-\lambda \sigma}\left[\frac{n \lambda \beta^{(2)}+\lambda \beta \sigma}{2}-\frac{\sigma}{2 n}+\frac{\sigma}{n^{2}} \sum_{j=1}^{n} y_{j}\right]$

where

$$
\sum_{j=1}^{n} y_{j}=n-\frac{1}{b}+\frac{n}{b} \cdot \frac{1-\gamma}{1-\gamma^{n}}
$$

with

$$
\begin{aligned}
\gamma_{F N, F Y, P N} & =1-n b \\
\gamma_{P Y} & =\frac{1-(n-1) b}{1+b}
\end{aligned}
$$

and $b>0$ is given in (28)-(31). [See the Appendix for the derivation of (34)-(36).] After some algebraic manipulation cf. (3)-(14), (16), we get the expected minipacket waiting times for the following diverse cases:

$$
\begin{aligned}
E W_{F N}= & \frac{\sigma}{n(2-(n+3) \lambda \sigma)} \\
& \cdot\left[\frac{(n+1)(n+2) \lambda \sigma}{3}-1+\frac{2}{n} \sum_{j=1}^{n} y_{j}^{F N}\right] \\
E W_{P N}= & \frac{\sigma}{n(2-(n+2) \lambda \sigma)} \\
& \cdot\left[\frac{n(n+1) \lambda \sigma}{3}-1+\frac{2}{n} \sum_{j=1}^{n} y_{j}^{P N}\right]
\end{aligned}
$$

$$
\begin{aligned}
E W_{F Y}= & \frac{\sigma}{n(2-(n+1) \lambda \sigma)} \\
& \cdot\left[\frac{(n-1)(n+1) \lambda \sigma}{3}-1+\frac{2}{n} \sum_{j=1}^{n} y_{j}^{F Y}\right] \\
E W_{P Y}= & \frac{\sigma}{n(2-n \lambda \sigma)} \\
& \cdot\left[\frac{n(n-2) \lambda \sigma}{3}-1+\frac{2}{n} \sum_{j=1}^{n} y_{j}^{P Y}\right] .
\end{aligned}
$$

\section{The StABILITy CONDITION AND THE High- AND LOW-LOAD EXPECTED DELAYS: SINGLE-SLOT RING}

Let us now consider approximations to the model for high and low load, as well as the stability condition. Cyclic and random polling are used to obtain the expected delays denoted by $E W^{\text {low }}$ and $E W^{\text {high }}$, respectively.

\section{A. Low-Load Case}

Cyclic polling is what happens when all the stations are empty. So, $E W^{\text {low }}$ could be a good estimate of the expected delay under low load. Note that $E W^{\text {low }}$ the estimates are also the lower bounds on the delays, since the expected delays can never be lower than that.

We take the cyclic service model in which a single-server $S$ attends the queues in a cyclic order: $Q_{1}, \cdots, Q_{n}$. The interarrival process, the service process and the switchover process are assumed to be mutually independent. We could use previously derived results for the cyclic server system, e.g., [3]. However, to obtain $E W^{\text {low }}$ we start from the derivation of $E W$. So, $a=1, b=0$ in the cyclic server system, and particularly in (18) and (22). Note that $\psi$ is the probability that the queue to which the service is offered is not empty, leading again to (25) and (32) with $0<\psi<1$. Clearly, (19)-(21), (23) and (24), (26)-(31) do not apply to this situation. The Appendix derivation is valid up to (A.15), where $\sum y_{j}$ is derived taking $b=0$, see (A.23). Clearly, $E W$ equations (33), (37)-(40) can be used, and taking $\sum y_{j}$ from (A.23), we have

$$
-1+\frac{2}{n} \sum_{j=1}^{n} y_{j}=n
$$

which leads to the following:

$$
\begin{aligned}
& E W_{F N}^{\text {low }}=\frac{\sigma}{n(2-(n+3) \lambda \sigma)}\left[\frac{(n+1)(n+2) \lambda \sigma}{3}+n\right] \\
& E W_{P N}^{\text {low }}=\frac{\sigma}{n(2-(n+2) \lambda \sigma)}\left[\frac{n(n+1) \lambda \sigma}{3}+n\right] \\
& E W_{F Y}^{\text {low }}=\frac{\sigma}{n(2-(n+1) \lambda \sigma)}\left[\frac{(n-1)(n+1) \lambda \sigma}{3}+n\right] \\
& E W_{P Y}^{\text {low }}=\frac{\sigma}{n(2-n \lambda \sigma)}\left[\frac{n(n-2) \lambda \sigma}{3}+n\right] .
\end{aligned}
$$




\section{B. High-Load Case}

The random polling model [4] provides an approximation for the mean waiting time $E W_{F}^{\text {high }}$ in Case $F$. The queues are attended by a single-server $S$, who randomly polls the queues; $S$ polls $Q_{i}$ at each polling instant with probability $1 / n$. We could use previously derived results for random polling systems, e.g., [4]. However, to obtain $E W_{F}^{\text {high }}$, we start from the derivation of $E W$. So, $b=1 / n$ in the random polling system, and for the full traffic case, (18) and (19) give $a=b=1 / n$. Note that $\psi$ is the probability that the queue to which the service is offered is not empty, leading again to (25) and (32), with $0<\psi<1$. Clearly, (20), (26), (28), and (30) do not apply to this situation. The derivation in the Appendix is valid, and $\sum y_{j}$ is derived taking $b=1 / n$ [see (A.22)]. Clearly, $E W$ equations (33), (37), and (39) can be used, and taking $\sum y_{j}$ from (A.22), we have

$$
-1+\frac{2}{n} \sum_{j=1}^{n} y_{j}=-1+\frac{2}{n} n^{2}=2 n-1
$$

which leads to the following

$$
\begin{aligned}
E W_{F N}^{\text {high }}= & \frac{\sigma}{n(2-(n+3) \lambda \sigma)} \\
& \cdot\left[\frac{(n+1)(n+2) \lambda \sigma}{3}+2 n-1\right] \\
E W_{F Y}^{\text {high }}= & \frac{\sigma}{n(2-(n+1) \lambda \sigma)} \\
& \cdot\left[\frac{(n-1)(n+1) \lambda \sigma}{3}+2 n-1\right] .
\end{aligned}
$$

Random polling, when applied to the model in Case $F$, leads to expected delays which are higher than the Markovian server routing results; it can easily be shown that $E W_{F}^{\text {high }}>E W_{F}$. Since the interarrival process, the service process, and the switchover process are mutually independent in these models, the $E W_{F}^{\text {high }}$ estimates do not provide a true upper bound on the delays in the rings. $E W$ could underestimate the delays in ATM-oriented rings, since it does not take into account mutual dependence between the service time and the polling process; therefore, $E W_{F}^{\text {high }}$ could be an approximation, especially under high loads, where the routing process resembles the random polling.

Random polling cannot be directly applied in Case $P$. Instead, taking $b_{P N}=1 /(n-1)$ and $a_{P Y}=b_{P Y}=1 /(n-1)$ in (18) and (22) leads to random polling of all queues but one at each polling instant. This further leads to a very complicated expression for an $E W_{P}^{\text {high }}$ and, in addition, the relation $E W_{P}^{\text {high }}>E W_{P}$ does not hold for all parameter values. So, we do not consider $E W_{P}^{\text {high }}$ in Case $P$ any further.

\section{Exact Stability Condition}

It has been shown (see, e.g., [4]) that the stability conditions of queues are the same for the random and for the cyclic polling systems. Clearly, the same stability condition holds for the Markovian server routing model (see [6]). Therefore, applied to the model of ATMR of the previous section, the stability condition is

$$
\frac{\lambda \sigma}{1-n \rho}<1
$$

Equation (49) can easily be derived from (A.14). Further, we derive that the upper bound $\lambda_{\text {sup }}$ on the load at $Q_{i}$ equals, cf. (4), (7), (10), (13), (15), and (49),

$$
\begin{aligned}
& \lambda_{\text {sup }}^{F N}=\frac{2}{(n+3) \sigma} \\
& \lambda_{\text {sup }}^{P N}=\frac{2}{(n+2) \sigma} \\
& \lambda_{\text {sup }}^{F Y}=\frac{2}{(n+1) \sigma} \\
& \lambda_{\text {sup }}^{P Y}=\frac{2}{n \sigma} .
\end{aligned}
$$

Note that the stability condition for the Orwell basic access mechanism (51) has already been obtained in [7], for Case $P N$.

\section{Approximate Modeling: Multiple-Slot Ring}

In this section, let us consider a multiple-slot ATMR. We have a multiple-server multiqueue polling model.

The multiple server model with $m$ cyclic servers can be approximated by a single cyclic server model having an $m$ times faster server. This has already been done in modeling of some other ATM-oriented rings [8], and for Orwell [9]. The simulation results show very small differences between the delays in the single- and multiple-slot case, as presented in Section VI (at most $6 \%$ for all loads and in all cases). An intuitive explanation for this is that the $m$ servers tend to cluster, appearing as a group of servers to the customers (intuitively, in large buildings elevators often stop at the same floor at the same time).

As applied to the ATM-oriented ring, this implies that an $m$-slot ring is approximated by a single-slot ring. All characteristics of the $m$-slot and the single-slot rings are the same including the same transmission rate, except for the distance between the successive stations, which is $m$-times smaller in the case of a single-slot ring as compared to the $m$-slot ring, i.e., the ring is $m$-times shorter.

Such an approximation leads to the exact stability condition. Note that the stability condition (49) is independent of the number of slots and that it also holds for a multiple-slot ring. Similarly, the high- and low-load case models can be used, and so (42)-(48) are used for a multiple-slot ring, as well.

Therefore, the same approximations for the mean waiting times are used for a single and for a multiple-slot ATMR in this paper. Note however, that the mean sojourn times, i.e., the mean times between the minipacket arrival at the source and its delivery at the destination must be quite different. This is due to the $m$ times longer propagation between the source and destination station in a multiple-slot ring as compared to a single-slot ring. This paper deals with mean waiting times estimates, and the mean sojourn times in ATMR's can be derived easily therefrom. 
TABLE I

Delays for Case $F$ with 30 Stations and a Single Slot

\begin{tabular}{c||c|c|c||c|c|c}
\hline \multirow{2}{*}{$\begin{array}{c}\text { relative } \\
\text { load }\end{array}$} & \multicolumn{3}{c||}{$\begin{array}{c}\text { N CASE } \\
\text { (max load }=251 \mathrm{Mbit} / \mathrm{s})\end{array}$} & \multicolumn{3}{c}{$\begin{array}{c}\text { Y CASE } \\
\text { (max load }=267 \mathrm{Mbit} / \mathrm{s})\end{array}$} \\
\cline { 2 - 7 } & $\begin{array}{c}\text { load } \\
{[\mathrm{Mbit} / \mathrm{s}]}\end{array}$ & $\begin{array}{c}\text { simulations } \\
\text { [slot] }\end{array}$ & $\begin{array}{c}\mathrm{EW} \\
\text { [slot] }\end{array}$ & $\begin{array}{c}\text { load } \\
\text { [Mbit/s] }\end{array}$ & $\begin{array}{c}\text { simulations } \\
\text { [slot] }\end{array}$ & $\begin{array}{c}\mathrm{EW} \\
\text { [slot] }\end{array}$ \\
\hline \hline 0.20 & 50 & $0.76 \pm 0.00$ & 0.76 & 53 & $0.76 \pm 0.00$ & 0.76 \\
\hline 0.40 & 100 & $1.26 \pm 0.00$ & 1.22 & 107 & $1.26 \pm 0.00$ & 1.22 \\
\hline 0.60 & 150 & $2.44 \pm 0.00$ & 2.24 & 160 & $2.46 \pm 0.00$ & 2.25 \\
\hline 0.80 & 200 & $6.87 \pm 0.00$ & 5.57 & 213 & $6.93 \pm 0.00$ & 5.56 \\
\hline 0.95 & 238 & $39.07 \pm 0.05$ & 25.44 & 253 & $38.67 \pm 0.04$ & 25.24 \\
\hline \hline
\end{tabular}

TABLE II

Delays for Case $F$ with Ten Stations

\begin{tabular}{|c|c|c|c|c|c|c|c|c|}
\hline \multirow{3}{*}{$\begin{array}{l}\text { relative } \\
\text { load }\end{array}$} & \multicolumn{4}{|c|}{$\begin{array}{c}\text { N CASE } \\
(\max \text { load }=212 \mathrm{Mbit} / \mathrm{s})\end{array}$} & \multicolumn{4}{|c|}{$\begin{array}{c}\text { Y CASE } \\
(\max \text { load }=251 \mathrm{Mbit} / \mathrm{s})\end{array}$} \\
\hline & \multirow{2}{*}{$\begin{array}{c}\text { load } \\
\text { [Mbit/s] }\end{array}$} & \multicolumn{2}{|c|}{ simulations [slot] } & \multirow{2}{*}{$\begin{array}{l}\text { EW } \\
\text { [slot] }\end{array}$} & \multirow{2}{*}{$\begin{array}{c}\text { load } \\
\text { [Mbit/s] }\end{array}$} & \multicolumn{2}{|c|}{ simulations [slot] } & \multirow{2}{*}{$\begin{array}{l}\text { EW } \\
\text { [slot] }\end{array}$} \\
\hline & & 1 slot & 30 slots & & & 1 slot & 30 slots & \\
\hline 0.20 & 42 & $0.74 \pm 0.00$ & $0.75 \pm 0.00$ & 0.75 & 50 & $0.74 \pm 0.00$ & $0.74 \pm 0.00$ & 0.75 \\
\hline 0.40 & 85 & $1.19 \pm 0.00$ & $1.23 \pm 0.00$ & 1.19 & 109 & $1.18 \pm 0.00$ & $1.21 \pm 0.00$ & 1.19 \\
\hline 0.60 & 127 & $2.18 \pm 0.00$ & $2.31 \pm 0.00$ & 2.16 & 150 & $2.18 \pm 0.00$ & $2.25 \pm 0.00$ & 2.15 \\
\hline 0.80 & 170 & $5.56 \pm 0.00$ & $5.91 \pm 0.00$ & 5.33 & 200 & $5.56 \pm 0.00$ & $5.66 \pm 0.00$ & 5.28 \\
\hline 0.95 & 201 & $27.94 \pm 0.04$ & $28.44 \pm 0.01$ & 24.75 & 238 & $27.48 \pm 0.04$ & $27.47 \pm 0.04$ & 24.12 \\
\hline
\end{tabular}

\section{Model AND Performance Evaluation}

\section{A. Test Runs}

A detailed simulation model of the ATM-oriented ring has been developed in Turbo Pascal 6.0. The analytic model has been tested by comparing the approximated expected minipacket waiting times to the results of simulations.

The following configuration, system parameters and workload have been considered:

1) Configuration: Transmission rate $=155 \mathrm{Mb} / \mathrm{s}$; number of slots $=1,30$; number of stations $=30,10,3$, and 2 ;

2) Slot Layout: Slot information field $=48 \mathrm{~B}$ and overhead in slot $=6 \mathrm{~B}$; and

3) Workload: Poisson arrivals of minipackets with relative load which is equal to $\lambda / \lambda_{\text {sup }}$.

The slot duration equals

$$
\sigma=2.787 \mu \mathrm{s}
$$

The full traffic matrix $(F)$, the partial traffic matrix $(P)$, as well as the slot reuse $(Y)$ and the nonreuse case $(N)$ were tested. The results of the simulations, as compared to the approximate analytic results, are given in Tables I-VI. The expected delays versus the load are illustrated in Figs. 3-6, where the simulation results, the analytically estimated ex-

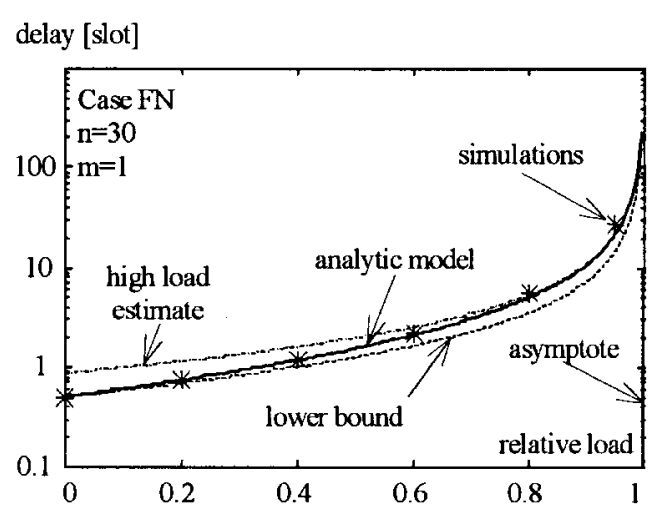

Fig. 3. The expected minipacket delays versus relative load per station in Case $F N$ with 30 stations and a single-slot ring.

pected delays, the high-load delay estimates, as well as the low bounds on delays, are shown. The minipacket delays are shown normalized to the slot duration $\sigma$.

The simulations have been quite lengthy, mostly $10^{9}$-slot duration or $7.5 \mathrm{~h}$ simulated time. $90 \%$ confidence intervals have been obtained. They are quite small in all experiments due to the lengthy runs. Halfwidth confidence intervals are less than 0.01 for the relative loads of up to 0.80 , and the largest halfwidth confidence interval is 0.08 for the relative load of 0.95 . 
TABLE III

Delays for CASE $F$ with Two Stations

\begin{tabular}{|c|c|c|c|c|c|c|c|c|}
\hline \multirow{3}{*}{$\begin{array}{l}\text { relative } \\
\text { load }\end{array}$} & \multicolumn{4}{|c|}{$\begin{array}{c}\text { N CASE } \\
(\max \operatorname{load}=110 \mathrm{Mbit} / \mathrm{s})\end{array}$} & \multicolumn{4}{|c|}{$\begin{array}{c}\text { Y CASE } \\
(\max \text { load }=184 \mathrm{Mbit} / \mathrm{s})\end{array}$} \\
\hline & \multirow{2}{*}{$\begin{array}{c}\text { load } \\
\text { [Mbit/s] }\end{array}$} & \multicolumn{2}{|c|}{ simulations [slot] } & \multirow{2}{*}{$\begin{array}{l}\text { EW } \\
\text { [slot] } \\
\end{array}$} & \multirow{2}{*}{$\begin{array}{c}\text { load } \\
\text { [Mbit/s] }\end{array}$} & \multicolumn{2}{|c|}{ simulations [slot] } & \multirow{2}{*}{$\begin{array}{l}\text { EW } \\
\text { [slot] }\end{array}$} \\
\hline & & 1 slot & 30 slots & & & 1 slot & 30 slots & \\
\hline 0.20 & 22 & $0.73 \pm 0.00$ & $0.71 \pm 0.00$ & 0.74 & 37 & $0.67 \pm 0.00$ & $0.67 \pm 0.00$ & 0.69 \\
\hline 0.40 & 44 & $1.10 \pm 0.00$ & $1.08 \pm 0.00$ & 1.15 & 73 & $0.95 \pm_{0.00}$ & $0.96 \pm 0.00$ & 1.02 \\
\hline 0.60 & 66 & $1.85 \pm 0.00$ & $1.88 \pm 0.00$ & 1.99 & 110 & $1.50 \pm 0.00$ & $1.54 \pm 0.00$ & 1.71 \\
\hline 0.80 & 88 & $4.10 \pm 0.00$ & $4.33 \pm 0.00$ & 4.66 & 147 & $3.17 \pm 0.00$ & $3.30 \pm 0.00$ & 3.88 \\
\hline 0.95 & 105 & $17.63 \pm 0.07$ & $18.28 \pm 0.06$ & 21.56 & 175 & $13.05 \pm 0.06$ & $13.56 \pm 0.04$ & 17.48 \\
\hline
\end{tabular}

TABLE IV

Delays for Case $P$ with 30 Stations and a Single Slot

\begin{tabular}{c||c|c|c||c|c|c}
\hline \hline \multirow{2}{*}{$\begin{array}{c}\text { relative } \\
\text { load }\end{array}$} & \multicolumn{3}{c||}{$\begin{array}{c}\text { N CASE } \\
\text { (max load }=258 \mathrm{Mbit} / \mathrm{s})\end{array}$} \\
\cline { 2 - 7 } & $\begin{array}{c}\text { load } \\
\text { [Mbit/s] }\end{array}$ & $\begin{array}{c}\text { simulations } \\
\text { [slot] }\end{array}$ & $\begin{array}{c}\mathrm{EW} \\
\text { [slot] }\end{array}$ & $\begin{array}{c}\text { load } \\
\text { [Mbit/s] }\end{array}$ & $\begin{array}{c}\text { simulations } \\
\text { [slot] }\end{array}$ & $\begin{array}{c}\mathrm{EW} \\
\text { [slot] }\end{array}$ \\
\hline \hline 0.20 & 52 & $0.76 \pm 0.00$ & 0.76 & 55 & $0.76 \pm 0.00$ & 0.76 \\
\hline 0.40 & 103 & $1.26 \pm 0.00$ & 1.22 & 110 & $1.27 \pm 0.00$ & 1.22 \\
\hline 0.60 & 155 & $2.47 \pm 0.00$ & 2.25 & 165 & $2.49 \pm 0.00$ & 2.25 \\
\hline 0.80 & 207 & $6.96 \pm 0.00$ & 5.57 & 220 & $7.01 \pm 0.00$ & 5.53 \\
\hline 0.95 & 245 & $39.21 \pm 0.04$ & 25.28 & 262 & $39.03 \pm 0.05$ & 24.71 \\
\hline \hline
\end{tabular}

TABLE $\mathrm{V}$

Delays for Case $P$ with Ten Stations

\begin{tabular}{|c|c|c|c|c|c|c|c|c|}
\hline \multirow{3}{*}{$\begin{array}{l}\text { relative } \\
\text { load }\end{array}$} & \multicolumn{4}{|c|}{$\begin{array}{c}\text { N CASE } \\
(\max \text { load }=230 \mathrm{Mbit} / \mathrm{s})\end{array}$} & \multicolumn{4}{|c|}{$\begin{array}{c}\text { Y CASE } \\
(\max \text { load }=276 \mathrm{Mbit} / \mathrm{s})\end{array}$} \\
\hline & \multirow{2}{*}{$\begin{array}{c}\text { load } \\
\text { [Mbit/s] }\end{array}$} & \multicolumn{2}{|c|}{ simulations [slot] } & \multirow{2}{*}{$\begin{array}{l}\text { EW } \\
\text { [slot] }\end{array}$} & \multirow{2}{*}{$\begin{array}{l}\text { load } \\
{[\mathrm{Mbit} / \mathrm{s}]}\end{array}$} & \multicolumn{2}{|c|}{ simulations [slot] } & \multirow{2}{*}{$\begin{array}{l}\text { EW } \\
\text { [slot] }\end{array}$} \\
\hline & & 1 slot & 30 slots & & & 1 slot & 30 slots & \\
\hline 0.20 & 46 & $0.74 \pm 0.00$ & $0.75 \pm 0.00$ & 0.75 & 55 & $0.74 \pm 0.00$ & $0.74 \pm 0.00$ & 0.74 \\
\hline 0.40 & 92 & $1.19 \pm 0.00$ & $1.22 \pm 0.00$ & 1.20 & 110 & $1.19 \pm 0.00$ & $1.20 \pm 0.00$ & 1.18 \\
\hline 0.60 & 138 & $2.22 \pm 0.00$ & $2.28 \pm 0.00$ & 2.18 & 165 & $2.20 \pm 0.00$ & $2.21 \pm 0.00$ & 2.13 \\
\hline 0.80 & 184 & $5.73 \pm 0.00$ & $5.83 \pm 0.00$ & 5.36 & 220 & $5.59 \pm 0.00$ & $5.52 \pm 0.00$ & 5.08 \\
\hline 0.95 & 218 & $28.92 \pm 0.05$ & $28.71 \pm 0.02$ & 24.44 & 262 & $27.41 \pm 0.04$ & $26.95 \pm 0.03$ & 22.18 \\
\hline
\end{tabular}

The maximum load a ring can carry is presented in the first row of a table. This load equals

$$
\begin{aligned}
& \max \_l o a d[b / s] \\
& =n \cdot \lambda_{\text {sup }}[\text { minipacket } / \mathrm{s}] \\
& \text { - slot_information_field_length[b/minipacket]. }
\end{aligned}
$$

The maximum load is independent of the number of slots, as is $\lambda_{\text {sup }}$, see (50)-(53). In addition, the maximum load in Case $P Y$ is independent of the number of stations and it equals 276 $\mathrm{Mb} / \mathrm{s}$ in all the numerical examples.
The ring with $Y$ option has a higher maximum load than the one with an $N$ option, with other parameters being the same (i.e., $\lambda_{\text {sup }}^{N}<\lambda_{\text {sup }}^{Y}$ since one switchover time is saved). Therefore, the relative load, e.g., 0.80, corresponds to different loads expressed in $\mathrm{Mb} / \mathrm{s}$ for $Y$ and $N$ options in all tables. For each relative load the $Y$ option is more efficient carrying a higher load.

We have in the limit, see (50)-(53)

$$
n \lambda_{\text {sup }} \rightarrow \frac{2}{\sigma}, \quad \text { if } n \rightarrow \infty
$$


TABLE VI

Delays for Case $P$ with Three Stations

\begin{tabular}{|c|c|c|c|c|c|c|c|c|}
\hline \multirow{3}{*}{$\begin{array}{l}\text { relative } \\
\text { load }\end{array}$} & \multicolumn{4}{|c|}{$\begin{array}{c}\text { N CASE } \\
(\max \operatorname{load}=165 \mathrm{Mbit} / \mathrm{s})\end{array}$} & \multicolumn{4}{|c|}{$\begin{array}{c}\text { Y CASE } \\
(\max l o a d=276 \mathrm{Mbit} / \mathrm{s}) \\
\end{array}$} \\
\hline & \multirow{2}{*}{$\begin{array}{c}\text { load } \\
\text { [Mbit/s] }\end{array}$} & \multicolumn{2}{|c|}{ simulations [slot] } & \multirow{2}{*}{$\begin{array}{l}\text { EW } \\
\text { [slot] }\end{array}$} & \multirow{2}{*}{$\begin{array}{c}\text { load } \\
\text { [Mbit/s] }\end{array}$} & \multicolumn{2}{|c|}{ simulations [slot] } & \multirow{2}{*}{$\begin{array}{l}\text { EW } \\
\text { [slot] } \\
\end{array}$} \\
\hline & & 1 slot & 30 slots & & & 1 slot & 30 slots & \\
\hline 0.20 & 33 & $0.70 \pm 0.00$ & $0.71 \pm 0.00$ & 0.73 & 55 & $0.67 \pm 0.00$ & $0.67 \pm 0.00$ & 0.68 \\
\hline 0.40 & 66 & $1.06 \pm 0.00$ & $1.08 \pm 0.00$ & 1.15 & 110 & $0.96 \pm 0.00$ & $0.96 \pm 0.00$ & 0.99 \\
\hline 0.60 & 99 & $1.81 \pm 0.00$ & $1.88 \pm 0.00$ & 2.06 & 165 & $1.55 \pm 0.00$ & $1.54 \pm 0.00$ & 1.61 \\
\hline 0.80 & 132 & $4.23 \pm 0.00$ & $4.38 \pm 0.00$ & 5.10 & 220 & $3.34 \pm 0.00$ & $3.31 \pm 0.00$ & 3.45 \\
\hline 0.95 & 157 & $20.10^{ \pm 0.08}$ & $19.84 \pm 0.04$ & 22.70 & 262 & $14.15^{ \pm} 0.05$ & $14.01 \pm 0.03$ & 14.32 \\
\hline
\end{tabular}

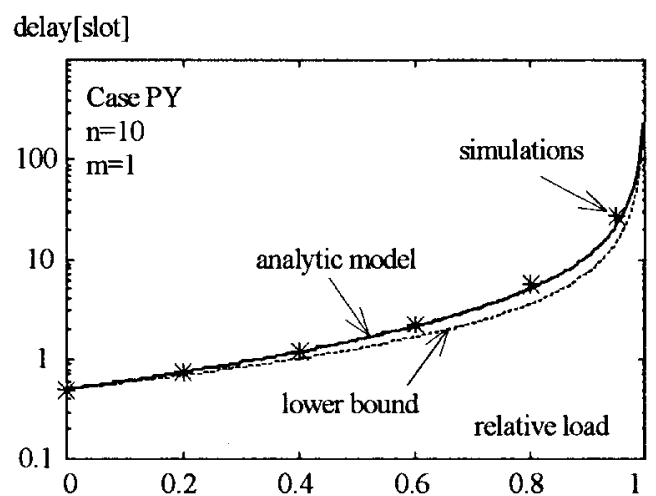

Fig. 4. The expected minipacket delays versus relative load per station in Case $P Y$ with ten stations and a single-slot ring.

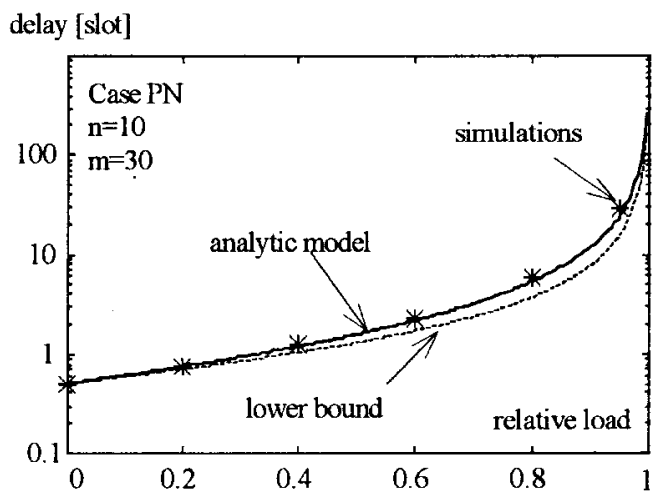

Fig. 5. The expected minipacket delays versus relative load per station in Case $P N$ with ten stations and 30 slots in the ring.

Because of (54)-(56), and since the slot information field length is $48 \mathrm{~B}$,

$$
\max \_ \text {load } \rightarrow 276 \mathrm{Mb} / \mathrm{s}, \quad \text { if } n \rightarrow \infty \text {. }
$$

Therefore, for large $n$, the maximum load would not be much different in all cases: $N$ and $Y, F$ and $P$. Interestingly, even the delays as obtained by the simulations are reasonably similar if $n=30$ in Case $F$ and also in Case $P$ (the difference between $Y$ and $N$ options is less than $1 \%$ in Tables I and IV).

The simulations delays of a single-slot ring and a 30-slot ring differ at most $6 \%$ for all loads and in all cases in Tables II,

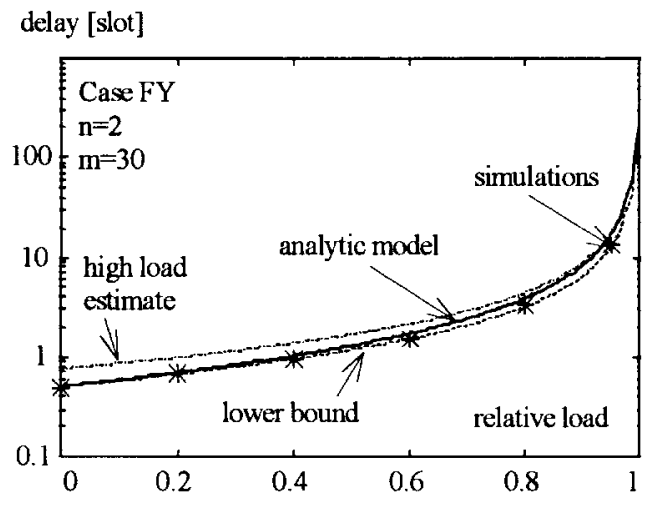

Fig. 6. The expected minipacket delays versus relative load per station in Case $F Y$ with two stations and 30 slots in the ring.

III, V, and VI. The smallest difference is in Case PY, at most $2 \%$, and the largest in Case FN, at most 6\%. The largest difference for the relative load 0.95 in all cases is $4 \%$. Note again that such a small difference in the delays was also an argument for us to use the developed single-slot model to approximate the delays in a multiple-slot ring.

Let us now consider the mean sojourn time in a multiple-slot ring by means of an example: Case $P N$ and a 30-slot ring. The mean minipacket propagation time between the source and the destination station equals $\tau / 2$, i.e., the expected minipacket service time $\beta_{P N}$ (7), which is the 15 -slot duration in the example. In the real ring, the destination station introduces an additional delay of a single-slot duration which is needed for the minipacket to be completely received. The expected minipacket waiting times in the example (see Tables $\mathrm{V}$ and VI) are smaller than 6-slot duration for relative loads of up to 0.80 . Clearly, the expected minipacket waiting times in the multiple-slot ring of the example are much smaller than the propagation time between the source and the destination for relative loads of up to 0.80 .

\section{B. The Model Accuracy}

The analytic model for a single-slot case (Tables I-VI, Figs. 3 and 4) gives an estimate of the expected minipacket waiting time which differs from the simulations as follows. 
1) For relative loads of up to 0.60 , the difference is less than 0.24 -slot duration or $0.7 \mu \mathrm{s}$ and the estimate deviates from the simulations results by less than $10 \%$.

2) For the relative load 0.80 the difference is less than 1.5slot duration or $4.2 \mu \mathrm{s}$ and the estimate deviates from the simulations results by less than $21 \%$.

The estimates almost equally differ from the simulations for a single-slot ring in all cases $F$ and $P, Y$ and $N$, even though Table IV, Case PY, has the maximum recorded difference.

The analytic model for a multiple-slot case (Tables II, III, V, and VI, Figs. 5 and 6) gives an estimate of the expected minipacket waiting time which differs from the simulations as follows.

1) For relative loads of up to 0.60 the difference is less than 0.15 -slot duration or $0.4 \mu \mathrm{s}$ and the estimate deviates from the simulations results by less than $7 \%$.

2) For the relative load 0.80 the difference is less than 0.6slot duration or $1.6 \mu \mathrm{s}$ and the estimate deviates from the simulations results by less than $10 \%$.

The estimates almost equally differ from the simulations for a multiple-slot ring in all cases $F$ and $P, N$ and $Y$, even though Table II, Case FN, has the maximum recorded difference.

For very high relative loads in excess of 0.80 , the analytic model has large deviations from the simulations: up to $40 \%$ for the 0.95 relative load. The model is not applicable for such loads.

The high and low-load estimates are useful since they can be compared to the simulations, to indicate the influence of the polling strategy on the delays. At very high loads, the simulations show delays which are sometimes even higher than $E W_{F}^{\text {high }}$, e.g., see Fig. 3. Since $E W_{F}^{\text {high }}$ also gives the upper bound on the analytic results obtained from the Markovian server routing model, the difference between the simulations and $E W_{F}^{\text {high }}$ and $E W$ shows the very high influence of the correlation between the service-time process and the polling process at very high loads. To significantly improve the analytic results, one has to include the mutual dependence between the polling and service processes (i.e., to remove the only approximation of this paper in the modeling of a single-slot ring).

According to the analytic estimates, the mean delays for Case $Y$ are smaller than those for Case $N$, i.e., $E W_{Y} \leq E W_{N}$ in all the numerical examples, given the relative load (with an exception of Table I, the relative load 0.80). According to the simulations, this relation also holds with an exception in the 30 -station cases with relative loads of up to 0.80 (Tables I and IV). Note again that the only explanation for analytic model inaccuracy is the dependence between the service time and the polling processes, which is not included in the model.

\section{Comparison to Other Models}

Let us compare the Markovian routing model to the other models of the basic access mechanism in ATM-oriented rings.

An approximate expression for mean message sojourn times in Case $P N$ is obtained using an approximate queueing model which was not a polling model but a single server, single queue with preemptive service discipline in [7]. That queueing model assumes Poisson batch arrivals of minipackets, and it estimates the message, not the minipacket, sojourn time and therefore cannot be compared to the model presented here.

The analytic model of [9] was also developed for Case PN. Our analytic model clearly has better numerical results than [9], which is usable for relative loads of up to 0.60 , and at that relative load it obtains up to $20 \%$ accuracy of the estimate. In addition, the Markovian server polling model result, e.g., [6], was not used in [9], and so it includes an approximate approach, two approximate formulas, and the solution is not given in a closed form. The mutual dependence between the polling and service processes has not been taken into account either. Hence, our approach leads to a superior prediction for an arbitrary problem, as compared to [9].

\section{CONCLUSION}

An approximate model which estimates the expected minipacket delay in the ATM-oriented ring with the destination release of used slots, has been developed in this paper. It is based on the multiqueue single-server Markovian server routing model.

The ATM-oriented ring is modeled in two protocol variants (Cases $Y$ and $N$ ): with and without the possibility of slot reuse by the destination station. The workload is fully symmetric, i.e., equal for all stations. Two traffic matrices are considered (Cases $F$ and $P$ ): a full traffic matrix where each station sends to every station including itself, and a partial traffic matrix where each station sends to all stations but itself.

The approximate analytic model provides estimates for the expected minipacket waiting times in a single-slot ring, (37)-(41). However, it can be applied to the multiple-slot ring as well, as the numerical examples show (up to 6\% difference in the delays in a single- and a 30-slot ring under any load). This effect is due to clustering of the servers resulting in a virtually single-server system with a powerful server. Interestingly, the expected minipacket waiting times can be much smaller than the mean minipacket sojourn times in a multiple-slot ring, due to propagation from the source to the destination station.

The analytic model was tested by comparing it to simulations. The expected minipacket waiting time estimates are very accurate for relative loads of up to 0.60 , leading to a difference of less than 0.24-slot duration, and the estimate deviates from the simulation results by less than $10 \%$, i.e., $0.7 \mu$ s. For relative loads of 0.80 the difference is less than 1.5-slot duration and the estimate deviates from the simulation results by less than $21 \%$, i.e., $4.2 \mu$ s. The model is not intended to be used for relative loads higher than 0.80 ; e.g., for relative loads of 0.95 , the difference is up to $40 \%$.

At very high loads, the simulations show very high delays, which are even higher than the analytic results obtained from the Markovian server routing model can provide $\left(E W_{F}^{\text {high }}\right.$ in Case $F$ ). To significantly improve the analytic results, one has to include the dependence between the polling and service processes, i.e., to remove the major approximation of this paper when modeling a single-slot ring. 
The exact stability conditions derived for ATMR's in (49)-(53) are independent of the number of slots in the ring. They lead to the expression for the maximum load a ring can carry (55). The ring with an $Y$ option has a higher maximum load than the one with an $N$ option. For each relative load the $Y$ option is more efficient carrying a higher load. However, the maximum load converges to the same value $(276 \mathrm{Mb} / \mathrm{s}$ in the numerical examples) as $n \rightarrow \infty$, all the parameters being the same and the number of slots arbitrary, and therefore the maximum load the ring can carry would not be much different for a large number of stations in all cases: $N$ and $Y, F$ and $P$.

\section{APPENDIX}

\section{ThE SERVER INTERVISIT TIMES}

Note that the equilibrium distribution of the polling probability $q_{i}$ for queue $Q_{i}$ in all cases: $F$ and $P, N$ and $Y$, equals

$$
q_{i}=1 / n \text {. }
$$

The expected time between the arrival of the server at $Q_{i}$ and its subsequent departure from $Q_{i}$ equals [6, Relation (5.29)]

$$
E V_{i}=\frac{1}{q_{i}} \cdot \frac{\rho \theta}{1-n \rho} \text {. }
$$

The expected time $f_{i}$ between the arrival of the server at $Q_{i}$ and its subsequent arrival at the next queue polled equals

$$
f_{i}=E V_{i}+\theta \text {. }
$$

Combining (A.1)-(A.3), we obtain

$$
f_{i}=\frac{\sigma}{n(1-n \rho)}
$$

Let us now evaluate $E T_{k i}$. According to [6, Lemma 5.1]

$$
E T_{k i}=f_{i}\left[1+\frac{1}{n} \sum_{l \neq k}\left(x_{i k}+x_{k l}-x_{i l}\right)\right]
$$

with

$$
x_{i j}:=E\{\# \text { steps required for the first entrance into }
$$

$$
\left.Q_{j} \text { starting from } Q_{i}\right\} \text {. }
$$

After a few algebraic manipulations, (A.5) becomes

$$
\begin{aligned}
\sum_{k \neq i} E T_{k i}=f_{i}\left\{(n-1)+\frac{1}{n}\right. & {\left[\sum_{k \neq i} \sum_{l \neq k} x_{i k}+\sum_{k \neq i} \sum_{l \neq k} x_{k l}\right.} \\
& \left.\left.-\sum_{k \neq i} \sum_{l \neq k} x_{i l}\right]\right\}
\end{aligned}
$$

It can easily be obtained that

$$
\begin{aligned}
& \sum_{k \neq i} \sum_{l \neq k} x_{i k}=(n-1) \sum_{k=1}^{n} x_{i k}-(n-1) x_{i i} \\
& \sum_{k \neq i} \sum_{l \neq k} x_{k l}=\sum_{k=1}^{n} \sum_{l=1}^{n} x_{k l}-\sum_{l=1}^{n} x_{i l}-\sum_{k=1}^{n} x_{k k}+x_{i i}
\end{aligned}
$$

$$
\sum_{k \neq i} \sum_{l \neq k} x_{i l}=(n-2) \sum_{l=1}^{n} x_{i l}+x_{i i}
$$

Placing (A.4) and (A.7)-(A.9) into (A.6), we receive

$$
\sum_{k \neq i} E T_{k i}=\frac{\sigma}{n(1-n \rho)}\left[\sum_{j=1}^{n} y_{j}-n\right]
$$

where

$$
\begin{aligned}
y_{1} & =x_{k+1, k} \cdots \\
y_{i} & =x_{k+i, k} \cdots \\
y_{n-1} & =x_{k-1, k} \\
y_{n} & =x_{k k} \\
k & =1, \cdots, n .
\end{aligned}
$$

In addition, using (A.1), it can easily be shown that

$$
y_{n}=x_{i i}=\frac{1}{q_{i}}=n \text {. }
$$

Note now that $E T_{i i}$, the expected time between two successive visits (and potential services) of the server at $Q_{i}$ equals

$$
E T_{i i}=x_{i i} f_{i}
$$

and placing (A.4) and (A.12) into (A.13)

$$
E T_{i i}=\frac{\sigma}{1-n \rho} \text {. }
$$

Let us, from now on, consider Cases $F$ and $P N$. From the theory of Markov chains, because of (18), we have

$\left[\begin{array}{c}y_{1} \\ y_{2} \\ \cdots \\ y_{i} \\ \cdots \\ y_{n-1} \\ y_{n}\end{array}\right]=\left[\begin{array}{c}1 \\ 1 \\ \cdots \\ 1 \\ \cdots \\ 1 \\ 1\end{array}\right]+\left[\begin{array}{cccccccc}b & b & b & \cdots & b & \cdots & b & 0 \\ a & b & b & \cdots & b & \cdots & b & 0 \\ b & b & b & \cdots & b & \cdots & b & 0 \\ b & b & b & \cdots & b & \cdots a & b & 0 \\ b & b & b & \cdots & b & \cdots & a & 0\end{array}\right]\left[\begin{array}{c}y_{1} \\ y_{2} \\ \cdots \\ y_{i} \\ \cdots \\ y_{n-1} \\ y_{n}\end{array}\right]$.

The first equation of (A.15) and (A.12) lead to

$$
\sum_{j=1}^{n} y_{j}=n+\frac{1}{b}\left(y_{1}-1\right), \quad b>0 .
$$

After a few algebraic manipulations from (A.12) and (A.15), it can be shown that

$$
y_{1}=n \frac{1-\gamma}{1-\gamma^{n}}
$$

with

$$
\gamma=a-b
$$

or, because of (19)

$$
\gamma_{F N, F Y, P N}=1-n b .
$$

Further, from (A.16) and (A.17), we get

$$
\sum_{j=1}^{n} y_{j}=n-\frac{1}{b}+\frac{n}{b} \cdot \frac{1-\gamma}{1-\gamma^{n}} .
$$


$\sum y_{j}$ in Case $F$ has a simple form, which can be obtained placing (26) and (A.19) into (A.20), and so

$$
\sum_{j=1}^{n} y_{j}^{F}=n\left[\frac{n}{1-\left(1-\psi_{F}\right)^{n}}-\frac{1-\psi_{F}}{\psi_{F}}\right] .
$$

Note that a random polling system can be represented by Case $F$ with $b=1 / n$. Then, we have from (A.19) and (A.20)

$$
\sum_{j=1}^{n} y_{j}=n^{2}, \quad b=\frac{1}{n} \text {. }
$$

If $b=0$, i.e., in a cyclic polling system, starting from (A.12) and (A.15), it can be shown that

$$
\sum_{j=1}^{n} y_{j}=\frac{n(n+1)}{2}, \quad b=0 .
$$

Let us consider Case PY in the sequel. From the theory of Markov chains, because of (22), we have

$\left[\begin{array}{c}y_{1} \\ y_{2} \\ \cdots \\ y_{i} \\ \cdots \\ y_{n-1} \\ y_{n}\end{array}\right]=\left[\begin{array}{c}1 \\ 1 \\ \cdots \\ 1 \\ \cdots \\ 1 \\ 1\end{array}\right]+\left[\begin{array}{cccccccc}0 & b & b & \cdots & b & \cdots & b & 0 \\ a & 0 & b & \cdots & b & \cdots & b & 0 \\ b & b & b & \cdots & & & & \\ b & & & \cdots & & \cdots & b & 0 \\ b & b & b & \cdots & b & . . a & 0 & 0 \\ b & b & b & \cdots & b & \cdots & a & 0\end{array}\right]\left[\begin{array}{c}y_{1} \\ y_{2} \\ \cdots \\ y_{i} \\ \cdots \\ y_{n-1} \\ y_{n}\end{array}\right]$.

The first equation of (A.24), and (A.12), lead to

$$
\sum_{j=1}^{n} y_{j}=n+\frac{1+b}{b} y_{1}-\frac{1}{b}, \quad b>0 .
$$

After some algebra from (A.12) and (A.24), it can be shown that

$$
y_{1}=\frac{n}{1+b} \cdot \frac{1-\gamma}{1-\gamma^{n}}
$$

with

$$
\gamma=\frac{a-b}{1+b}
$$

or, because of (23)

$$
\gamma_{P Y}=\frac{1-(n-1) b}{1+b} .
$$

Substituting (A.26) into (A.25), we obtain the relation (A.20), with $\gamma$ as given in (A.28).

\section{ACKNOWLEDGMENT}

The authors wish to thank the anonymous referees and Editor P. O'Reilly, for valuable comments.

\section{REFERENCES}

[1] K. Imai, T. Ito, H. Kasahara, and N. Morita, "ATMR: Asynchronous transfer mode ring protocol," Comp. Networks \& ISDN Syst., vol. 26, nos. 6-8, pp. 785-798, Mar. 1994.

[2] J. L. Adams, "Orwell," Comp. Networks \& ISDN Syst., vol. 26, nos. 6-8, pp. 771-784, Mar. 1994.

[3] H. Takagi, Analysis of Polling Systems. Cambridge, MA: MIT Press, 1986.

[4] L. Kleinrock and H. Levy, "The analysis of random polling systems," Oper. Res., vol. 36, no. 5, pp. 716-732, Sept./Oct. 1988.

[5] O. J. Boxma and W. P. Groenendijk, "Pseudoconservation laws in cyclic-service systems," J. Applicat. Prob., vol. 27, Dec. 1987.

[6] O. J. Boxma and J. A. Weststrate, "Waiting times in polling systems with Markovian server routing," in Proc. Messung, Modellierung und Bewertung von Rechensystemen und Netzen, G. Stiege and J. S. Lie, (Hrsg.) Braunschweig, Germany: Springer-Verlag, Sept. 1989.

[7] M. Zafirovic-Vukotic and I. G. Niemegeers, "Performance modeling of the Orwell basic access mechanism," in ACM SIGCOM'87, Comp. Comm. Rev., vol. 17, no. 5, pp. 35-48, 1987.

[8] _ "Performance modeling of a HSLAN slotted ring protocol," in ACM SIGMETRICS'88, Performance Eval. Rev., vol. 16, no. 1, pp. 37-46, 1988.

[9] B. van Arem and E. van Doorn, "Analysis of a queueing model for slotted ring networks," Comput. Networks \& ISDN Syst., vol. 20, pp. 309-314, 1990.

[10] TransXpress ALine, Siemens AS, Oslo, Norway, Product Folder, Tech Description, Product Release 1.0, A30808-X3693-B105-1-7618, Feb. 1998.

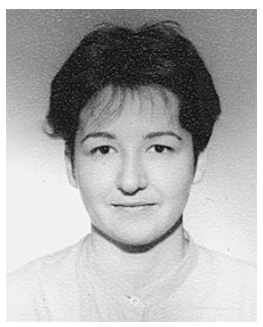

Mirjana Zafirovic-Vukotic (M'92-SM'97) received the B.Sc. degree in applied mathematics, with a minor in computer science, and the M.Sc. degree in operations research, from the University of Belgrade, Yugoslavia, in 1981 and 1984, respectively. She received the Ph.D. degree (cum laude) in technical sciences from the University of Twente, The Netherlands, in 1988.

She is currently a Research Staff Member at the M. Pupin Institute, Telecommunications Department, Yugoslavia, and an Associate Professor with the School of Electrical Engineering, University of Belgrade. Her research interests include protocol design and performance analysis for high speed communications.

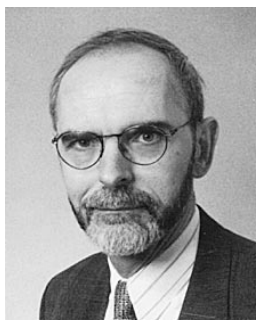

Ignatius G. M. M. Niemegeers (S'70-M'73) was born in Gent, Belgium, in 1947. He received the M.Sc. degree in electrical engineering from the Rijksuniversiteit Gent in 1970. He received the M.Sc. degree in computer engineering in 1972, and the $\mathrm{Ph} . \mathrm{D}$. degree from Purdue University, West Lafayette, IN, in 1978.

From 1978 to 1981 , he was with the Research Department of Bell Telephone Manufacturing Company, Antwerp, Belgium, where he was involved in the design of packet-switching systems. From 1981 to 1986, he was an Associate Professor in the Electrical Engineering Department, University of Twente, Enschede, The Netherlands. Since 1986, he has been a Full Professor holding the Communication Networks Chair in the Computer Science Department. He is currently a Scientific Director of the Center for Telematics and Information Technology, University of Twente, a multidisciplinary research institute focusing on ICT systems and their applications. 